\title{
Phototherapy and dithranol treatment of psoriasis: new lamps for old
}

\author{
P M FARR, B L DIFFEY, JANET M MARKS
}

\begin{abstract}
The response of psoriasis to ultraviolet radiation and dithranol was compared with the response to dithranol alone in 24 patients. The difference in rate of response, measured as change in plaque thickness, and the difference in time to complete clearance of psoriasis between irradiated and non-irradiated forearm lesions was significantly greater for patients treated using fluorescent lamps with negligible ultraviolet $\mathbf{C}$ emission (Wolff Helarium) than for those patients treated with a medium pressure mercury arc lamp $(p<0.01)$ or an array of fluorescent sunlamps $(p<0.05)$.

The difference in therapeutic response shows that ultraviolet $B$ phototherapy is effective when used in combination with dithranol. Nevertheless, radiation sources with substantial ultraviolet $\mathrm{C}$ emission, such as the medium pressure mercury arc lamp most commonly used to treat psoriasis in the United Kingdom, have little effect because delivery of therapeutic doses of ultraviolet $B$ is limited by erythema induced by ultraviolet $C$.
\end{abstract}

\section{Introduction}

Dithranol is the main drug used for the hospital treatment of psoriasis in the United Kingdom. It is usually given concomitantly with ultraviolet radiation phototherapy, which is time consuming, requires trained staff, and has short term and long term hazards.' Furthermore, studies have failed to show any additional therapeutic benefit of combined ultraviolet radiation and dithranol over dithranol alone, ${ }^{2-4}$ even though sunlight and other sources of ultraviolet radiation ${ }^{5}$ can improve psoriasis.

\footnotetext{
University Department of Dermatology, Royal Victoria Infirmary, Newcastle upon Tyne NE1 4LP

P M FARR, MRCP, senior registrar

JANET M MARKS, DM, FRCP, senior lecturer

Regional Medical Physics Department, Dryburn Hospital, Durham B L DIFFEY, PHD, FINSTP, top grade physicist

Correspondence to: Dr Farr.
}

To explain this apparently different therapeutic effect the role of phototherapy in the treatment of psoriasis with dithranol was examined in a controlled study using three ultraviolet lamps with different spectral emissions.

\section{Patients and methods}

We studied 24 patients aged 16-59 (11 male, 13 female) admitted to hospital for dithranol treatment of chronic plaque psoriasis. The only requirement for entry into the open trial was disease of the flexor aspects of both forearms, which allowed measurement of lesion thickness (see below). The effect of natural sunlight on the psoriasis (improved, no change, or worsened) and the sun reactive skin type were recorded in each case.

Dithranol treatment-The dermatologist admitting the patient decided whether dithranol was to be given using the Ingram ${ }^{6}$ or short contact ${ }^{78}$ regimen, which have equal therapeutic effect in psoriasis. ${ }^{9}$ Treatment was applied daily to all lesions until clearance of psoriasis was achieved (judged clinically) and the concentration of dithranol was increased to the limit of tolerance in each patient. Daily baths containing coal tar solution were part of the Ingram regimen but were not used in patients treated with short contact dithranol.

Phototherapy-Three radiation sources were used: (1) a medium pressure mercury arc lamp; (2) an array of six $60 \mathrm{~cm}$ fluorescent sunlamps (Philips type TL20W/12) housed in a solarium; (3) an array of six $60 \mathrm{~cm}$ ultraviolet fluorescent lamps (Wolff Helarium B1-01.40W) housed in a solarium. Each of the lamps was used to treat eight patients recruited consecutively. In each case the flexor aspect of one forearm was irradiated before the treatment with dithranol on six days each week. The exposure regimen was designed to achieve a similar degree of erythema in each patient irrespective of the radiation source. The initial exposure time was $70 \%$ of that required to produce minimal erythema, predicted from the sun reactive skin type for each patient. Daily increments in exposure time of $40 \%$ were employed until erythema developed, after which $0-20 \%$ increments were used, depending on the intensity of the erythemal response.

Assessment of response-Single spring Harpenden callipers ${ }^{10}$ were used to measure the difference in lesion thickness of symmetrical plaques of psoriasis on each forearm compared with adjacent normal skin. Measurements were made on the same areas of affected and unaffected skin before treatment was started and then on alternate days until the lesions on the forearm had regressed as assessed clinically by inspection and palpation.

Statistical analysis-For each patient regression analysis of the logarithm of plaque thickness was applied to calculate the rate of clearance $\left(\lambda\right.$,days $\left.{ }^{-1}\right)$ for both the irradiated and non-irradiated arms. The difference in response 
among the three treatment groups was examined using the Kruskal-Wallis test and the difference in response between each of the lamps examined using the Wilcoxon two sample test.

\section{Results}

The distribution of sun reactive skin types was similar for the three treatment groups (table I), 17 (71\%) of the patients being of skin type 2 . From the history natural sunlight improved the psoriasis in 14, worsened the was significantly greater for the patients treated with the Helarium lamps $\underline{7}$ than those treated with the fluorescent sunlamps $(p<0.05)$ or the medium 3 pressure mercury arc lamp $(p<0.01)$. There was no significant difference in $\stackrel{\mathbb{Q}}{\circ}$ response between the patients treated with the fluorescent sunlamps and the $c$ medium pressure mercury arc lamp.

The Kruskal-Wallis test showed that the differences in time to complete clearance of psoriasis (table III) between the irradiated and non-irradiated arms in each of the three treatment groups varied $(p<0.01)$. The reduction $\mathbb{D}$ in time to complete clearance was significantly greater for the patients treated with the Helarium lamps than those treated with the fluorescent 0 sunlamps $(p=0.05)$ or the medium pressure mercury $\operatorname{arclamp}(p<0.01)$ and

TABLE I-Distribution of sun reactive skin type, dithranol treatment, and effect of sunlight on psoriasis in the three groups of patients

\begin{tabular}{|c|c|c|c|c|c|c|c|c|}
\hline & \multicolumn{3}{|c|}{ Skin type } & \multicolumn{2}{|c|}{ Dithranol treatment } & \multicolumn{3}{|c|}{ Effect of sunlight } \\
\hline & $\begin{array}{c}1 \\
\text { (Always burns, } \\
\text { never tans) }\end{array}$ & $\begin{array}{c}2 \\
\text { (Always burns, } \\
\text { sometimes tans) }\end{array}$ & $\begin{array}{c}3 \\
\begin{array}{c}\text { (Sometimes burns, } \\
\text { always tans) }\end{array}\end{array}$ & Ingram & $\begin{array}{l}\text { Short } \\
\text { contact }\end{array}$ & $\begin{array}{l}\text { Psoriasis } \\
\text { improved }\end{array}$ & $\begin{array}{c}\text { No } \\
\text { change }\end{array}$ & $\begin{array}{l}\text { Psoriasis } \\
\text { worsened }\end{array}$ \\
\hline $\begin{array}{l}\text { Mercury arc lamp } \\
\text { Fluorescent sunlamp } \\
\text { Helarium lamp }\end{array}$ & $\begin{array}{l}0 \\
0 \\
1\end{array}$ & $\begin{array}{l}5 \\
6 \\
6\end{array}$ & $\begin{array}{l}3 \\
2 \\
1\end{array}$ & $\begin{array}{l}3 \\
2 \\
3\end{array}$ & $\begin{array}{l}5 \\
6 \\
5\end{array}$ & $\begin{array}{l}6 \\
4 \\
4\end{array}$ & $\begin{array}{l}0 \\
3 \\
3\end{array}$ & $\begin{array}{l}2 \\
1 \\
1\end{array}$ \\
\hline Totals & 1 & 17. & 6 & 8 & 16 & 14 & 6 & 4 \\
\hline
\end{tabular}

TABLE II-Time taken (in days) for thickness of psoriatic plaques to reach $50 \%$ of initial thickness estimated from regression analysis

\begin{tabular}{|c|c|c|c|c|c|c|c|c|}
\hline \multicolumn{3}{|c|}{ Mercury arc lamp } & \multicolumn{3}{|c|}{ Fluorescent sunlamp } & \multicolumn{3}{|c|}{ Helarium lamp } \\
\hline Subject & Control arm & Irradiated arm & Subject & Control arm & Irradiated arm & Subject & Control arm & Irradiated arm \\
\hline 1 & 5 & 6 & 9 & 2 & 3 & 17 & 17 & 8 \\
\hline 2 & 9 & 8 & 10 & 6 & 6 & 18 & 9 & 4 \\
\hline 3 & 9 & 7 & 11 & 14 & 6 & 19 & 8 & 3 \\
\hline 4 & 10 & 9 & 12 & 13 & 10 & 20 & 7 & 5 \\
\hline 5 & 5 & 5 & 13 & 12 & 14 & 21 & 11 & 4 \\
\hline 6 & 7 & 6 & 14 & 6 & 4 & 22 & 9 & 5 \\
\hline 7 & 5 & 6 & 15 & 12 & 8 & 23 & 9 & 6 \\
\hline 8 & 8 & 10 & 16 & 4 & 3 & 24 & 8 & 3 \\
\hline Median & $7 \cdot 5$ & 6.5 & & $9 \cdot 0$ & $6 \cdot 0$ & & $9 \cdot 0$ & $4 \cdot 5$ \\
\hline
\end{tabular}

TABLE III-Clearance time (in days) estimated clinically for the three groups of subjects

\begin{tabular}{|c|c|c|c|c|c|c|c|c|}
\hline \multicolumn{3}{|c|}{ Mercury arc lamp } & \multicolumn{3}{|c|}{ Fluorescent sunlamp } & \multicolumn{3}{|c|}{ Helarium lamp } \\
\hline Subject & Control arm & Irradiated arm & Subject & Control arm & Irradiated arm & Subject & Control arm & Irradiated arm \\
\hline 1 & 13 & 13 & 9 & 11 & 11 & 17 & 25 & 23 \\
\hline 2 & 13 & 13 & 10 & 17 & 14 & 18 & 18 & 13 \\
\hline 3 & 14 & 14 & 11 & 17 & 14 & 19 & 7 & 7 \\
\hline 4 & 18 & 18 & 12 & 18 & 18 & 20 & 18 & 14 \\
\hline 5 & $\begin{array}{r}.8 \\
.\end{array}$ & 10 & 13 & 22 & 22 & 21 & 19 & 14 \\
\hline 6 & 15 & 15 & 14 & 13 & 13 & 22 & 11 & 9 \\
\hline 7 & 12 & 13 & 15 & 20 & 17 & 23 & 16 & 12 \\
\hline 8 & 20 & 21 & 16 & 15 & 13 & 24 & 19 & 9 \\
\hline Median & 13.5 & $13 \cdot 5$ & & $17 \cdot 0$ & $14 \cdot 0$ & & $18 \cdot 0$ & $12 \cdot 5$ \\
\hline
\end{tabular}

psoriasis in four, and caused no change in the remainder. All patients completed the trial and were cleared of psoriasis, the Ingram regimen being used in eight patients and short contact dithranol in 16. There was no significant difference (Wilcoxon two sample test) in the rate of clearance or time to complete clearance of psoriasis between the non-irradiated arms of patients receiving the Ingram regimen or short contact dithranol. Moderate erythema on the irradiated forearm was seen in all patients on at least two separate occasions during the study.

Plaque thickness, expressed as a percentage of pretreatment measurements, decreased exponentially with the number of days of treatment. Table II shows the number of days of treatment required for a $50 \%$ reduction in plaque thickness $\left(\log ^{(2)} / \lambda\right)$, or half clearance time, for each patient. The Kruskal-Wallis test showed that the differences in half clearance time between the irradiated and non-irradiated arms in each of the three treatment groups varied $(p<0.01)$. The reduction in time to half clearance was significantly greater for the patients treated with the fluorescento sunlamps compared with those treated with the medium pressure mercurye $\operatorname{arc} \operatorname{lamp}(\mathrm{p}<0.05)$.

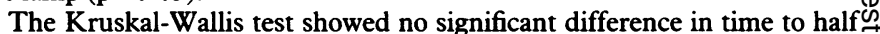
clearance (table II) or complete clearance (table III) of the non-irradiated 0 arms in each of the three groups.

\section{Discussion}

Our results show a significant difference in therapeutic response of psoriasis to combined ultraviolet radiation and dithranol that depended on the radiation source. The median time to halfo

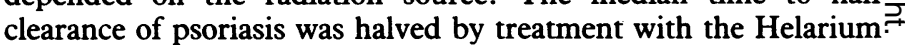


lamps, and this response was significantly greater than that obtained with the other two lamps. The median time to complete clearance of psoriasis (judged clinically) was reduced by $31 \%$ with the Helarium lamps, $18 \%$ with the fluorescent sunlamps, and was unchanged in the group treated with the medium pressure mercury arc lamp.
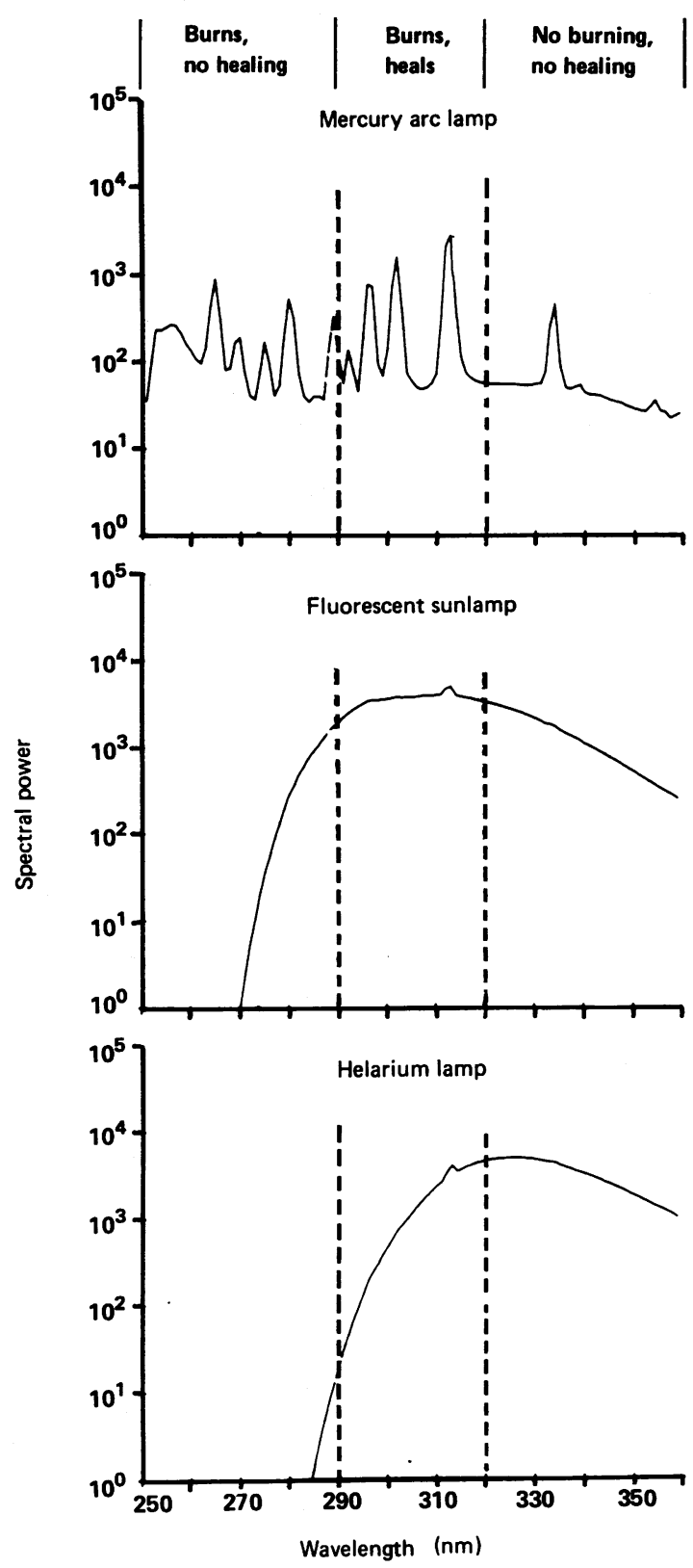

Spectral power (relative units) of the three lamps and effect on psoriasis of ultraviolet C $(<290 \mathrm{~nm})$, ultraviolet B $(290-320 \mathrm{~nm})$, and ultraviolet A $(320-400 \mathrm{~nm})$ radiation.

In each patient, irrespective of the radiation source, a similar degree of ultraviolet erythema was achieved that persisted for several days on at least two separate occasions during the study. Although a different therapeutic response would be expected had a more aggressive exposure regimen been employed, it is unlikely that patients would tolerate erythema of greater severity, especially if whole body irradiation was performed. Thus our results represent the maximum benefit that could be achieved in practice from each of the lamps. For each lamp there was no significant difference in response between the patients treated with the Ingram or short contact regimen, showing that the use of tar confers no additional benefit in the treatment of psoriasis with ultraviolet radiation and dithranol. As the three treatment groups were also similar with regard to distribution of sun reactive skin types and response of psoriasis to natural sunlight, the difference in therapeutic response that we found must depend on the radiation source.

All of the lamps emit ultraviolet A $(320-400 \mathrm{~nm})$ and ultraviolet B $(290-320 \mathrm{~nm})$ but they differ in the ultraviolet $C$ region $(200-290 \mathrm{~nm})$ (figure). In high doses ultraviolet A irradiation improves psoriasis, " but with the exposure times that we used the therapeutic effect of this component from each of the lamps can be discounted. Ultraviolet C wavelengths account for $66 \%$ of the erythemally effective power of the medium pressure mercury arc lamp, $26 \%$ of the fluorescent sunlamps, but only $0.9 \%$ of the Helarium lamp. Thus as the lamp that we found to be most effective had the least ultraviolet $C$ emission, its therapeutic effect must be due to ultraviolet $B$. Human skin is very sensitive to ultraviolet $C,,^{12}$ and in therapeutic use as in our study the erythema induced by these wavelengths would be an important limiting factor with regard to the maximum ultraviolet $B$ exposure dose possible with the medium pressure mercury arc lamp or fluorescent sunlamps.

Our findings show that even in erythemal doses there is little or no therapeutic response of dithranol treated psoriasis to lamps with substantial ultraviolet $\mathrm{C}$ emission, which is in keeping with the observation that these wavelengths are ineffective when phototherapy alone is used to treat psoriasis. ${ }^{13}$ The use of medium pressure mercury arc lamps explains why other studies failed to show any therapeutic benefit of phototherapy in the treatment of psoriasis with dithranol, ${ }^{24}$ despite the well known beneficial response to sunlight.

The two lamps that we found to have little or no therapeutic effect are representative of radiation sources commonly used to treat psoriasis. A recent survey (B L Diffey, A F McKinlay, unpublished) showed that most physiotherapy departments in the United Kingdom use the medium pressure mercury arc ("hot quartz") lamp-for example, the Alpine sunlamp, Kromayer lamp, Hanau 3002, Hohensonne 3030, Osram Ultravitalux, Nomeq sunlamp, Prescription lamp - for phototherapy of psoriasis. The fluorescent sunlamp-for example, Philips type TL20W/12 and Westinghouse FS sunlamp-commonly used in the United States and Europe is found in some phototherapy units in the United Kingdom-for example, the Theraktin UV bath. The Helarium fluorescent lamp, which we found to be most effective, has recently been developed specifically for the treatment of psoriasis.

Our results show for the first time that ultraviolet radiation phototherapy improves the treatment of psoriasis with dithranol; however, most ultraviolet lamps used in the United Kingdom for psoriasis have little effect because delivery of therapeutic doses of ultraviolet $\mathrm{B}$ is limited by erythema induced by ultraviolet $\mathrm{C}$. We therefore suggest that lamps that emit substantial amounts of ultraviolet $\mathrm{C}$ should be abandoned and be replaced by sources which, like terrestrial sunlight, have no ultraviolet $\mathrm{C}$ component.

We are grateful to Professor Sam Shuster for constructive criticism of the manuscript.

\section{References}

1 Marks JM. Adverse effects of ultraviolet light therapy. Adverse Drug Reaction Bulletin 1982 Aug:348-51.

2 Bowers RE, Dalton D, Fursdon D, Knowelden J. The treatment of psoriasis with UVR, dithranol paste and tar baths. Brf Dermatol 1966;78:273-81.

3 Young E. The external treatment of psoriasis. Br $\mathcal{O}$ Dermatol 1970;82:516-9.

4 Seville RH. Simplified dithranol treatment of psoriasis. Br D Dermatol 1975;93:205-8.

Le Vine MJ, White HAD, Parrish JA. Components of the Goeckerman regimen. $\mathcal{I}$ Invest Dermato 1979;73:170-3.

17gram JT. The significance and management of psoriasis. BrMed $\mathcal{f}$ 1954;ii:823-8.

7 Schaeffer H, Farber EM, Goldberg L, Schalla W. Limited application period for dithranol in psoriasis. Br $\mathcal{F}$ Dermatol 1980;102:571-3.

8 Runne U, Kunze J. Minutes treatment with anthralin in psoriasis. Br 7 Dermatol 1982;106:135-9.

9 Marsden JR, Copburn PR, Marks JM, Shuster S. Measurement of the response of psoriasis to short-term application of anthralin. Br $\mathcal{X}$ Dermatol 1983;109:209-18.

10 Rogers S, Chadkirk W, Comaish JS, Marks JM, Rawlins MD, Shuster S. Objective measurement of the response of psoriasis to treatment. $\mathcal{F}$ Invest Dermatol 1979;72:286.

1 Parrish JA. The treatment of psoriasis with longwave ultraviolet light (UV-A). Arch Dermato 1977;113:1525-8.

12 Farr PM, Diffey BL. The erythemal response of human skin to ultraviolet radiation. Brf Dermatol 1985;113:65-76.

13 Parrish JA, Jaenicke KF. Action spectrum for phototherapy of psoriasis. $\mathcal{J}$ Invest Dermatol 1981;76:359-62.

(Accepted 12 November 1986) 